\title{
PREVALENCE AND ANTIMICROBIAL SUSCEPTIBILITY PATTERN OF METHICILLIN RESISTANT STAPHYLOCOCCUS AUREUS (MRSA) ISOLATES IN A TERTIARY CARE HOSPITAL IN PUNJAB
}

\author{
Maninder Kaur ${ }^{1}$, Satish Gupte ${ }^{2}$, Prerna Aggarwal ${ }^{3}$, Ashwini Manhas ${ }^{4}$, Manju Bala5
}

\section{HOW TO CITE THIS ARTICLE:}

Maninder Kaur, Satish Gupte, Prerna Aggarwal, Ashwini Manhas, Manju Bala. "Prevalence and its antimicrobial susceptibility pattern of Methicillin Resistant staphylococcus aureus (MRSA) isolates in a tertiary care Hospital in Punjab". Journal of Evolution of Medical and Dental Sciences 2013; Vol. 2, Issue 41, October 14; Page: 78417846.

ABSTRACT: BACKGROUND: Methicillin resistant Staphylococcus aureus (MRSA) is an important cause of nosocomial infections worldwide. This prospective study was undertaken to know the prevalence of MRSA, to study antimicrobial susceptibility pattern and inducible clindamycin resistance in Staphylococcus aureus isolates obtained from the indoor patients of a tertiary care hospital in Punjab. MATERIALS AND METHODS: All the isolates of Staphylococcus aureus obtained from various clinical specimens were identified by standard methods. MRSA was detected using 30ug cefoxitin disc by disc diffusion method as per CLSI guidelines, 2007. Each isolate was tested for other anti-staphylococcal antibiotics by Kirby-Bauer disc diffusion method. Additionally, inducible clindamycin resistance was studied by disc induction test (D test). RESULTS: During a period of one year, a total of 252 isolates of Staphylococcus aureus were obtained. Eighty six (34.1\%) isolates were methicillin resistant Staphylococcus aureus (MRSA) and the remaining 166 (65.8\%) isolates were methicillin sensitive Staphylococcus aureus (MSSA). MRSA isolates were significantly less sensitive to common anti-staphylococcal antibiotics as compared to MSSA isolates. However, MRSA isolates showed relatively better sensitivity to some of the antibiotics like netilmicin, levofloxacin, tetracycline and clindamycin. Susceptibility to ciprofloxacin was low in both MSSA (19.8\%) and MRSA (9.3\%). None of the isolate of Staphylococcus aureus was resistant to vancomycin, linezolid and teicoplanin. Inducible clindamycin resistance was present in 17 (19.7\%) MRSA isolates as against 8 (4.8\%) MSSA isolates. CONCLUSION: Robust antimicrobial stewardship and strengthened infection control measures are required to prevent spread and reduce emergence of resistance.

KEYWORDS: Methicillin resistant Staphylococcus aureus, cefoxitin, antibiogram, inducible clindamycin resistance.

INTRODUCTION: Healthcare-associated methicillin resistant Staphylococcus aureus is a major cause of nosocomial infections worldwide, with significant attributable morbidity and mortality in addition to pronounced healthcare costs. ${ }^{1}$ Since MRSAs are resistant to all B -lactam antibiotics, the therapeutic options are limited significantly. ${ }^{2}$ Therefore, accurate detection of methicillin resistance in Staphylococcus aureus is of utmost importance to ensure effective treatment for the affected patient and to prevent further transmission. ${ }^{3}$

The gold standard for identifying MRSA is to detect the mecA gene, ${ }^{2}$ or its product, PBP2a, by latex agglutination. ${ }^{4}$ However, these tests are not within the scope of resource constrain setting and are relatively expensive. Cefoxitin, a cephamycin is a potent inducer of the mecA regulatory system. ${ }^{2}$ CLSI recommends usage of cefoxitin instead of oxacillin when using the disc diffusion method to 


\section{ORIGINAL ARTICLE}

determine resistance against methicillin for Staphylococcus aureus. ${ }^{5}$ Cefoxitin results are easier to interpret than those of oxacillin and are thus more sensitive for the detection of mecA-mediated resistance than oxacillin results. ${ }^{6,7}$

The epidemiology of MRSA is not uniform in different parts of India. The incidence of MRSA in India ranges from $30 \%-70 \%{ }^{8,9}$ Therefore, the present study has been carried out to find the prevalence of MRSA in a tertiary care hospital in Punjab, to study antimicrobial susceptibility pattern and inducible clindamycin resistance in Staphylococcus aureus.

MATERIALS AND METHODS: The study has been carried in the tertiary care hospital from March 2012 to March 2013. A total of 252 isolates of Staphylococcus aureus were obtained from various clinical specimens like pus, blood, urine, respiratory specimen and body fluids of the indoor patients. The isolates were identified biochemically by standard laboratory procedures. ${ }^{10}$ The antibiotic susceptibility test of all the confirmed isolates was performed on Mueller Hinton agar (Hi Media) using Kirby-Bauer method. A bacterial suspension with the turbidity adjusted to a 0.5 McFarland standard was used. The antibiotic discs used for sensitivity testing of isolates were: penicillin-G (10 $\mathrm{U})$, ampicillin $(10 \mu \mathrm{g})$, ciprofloxacin $(5 \mu \mathrm{g})$, levofloxacin $(5 \mu \mathrm{g})$, tetracycline (30 $\mu \mathrm{g})$, netilmicin $(30$ $\mu \mathrm{g})$, gentamicin $(10 \mu \mathrm{g})$, cefoxitin $(30 \mu \mathrm{g})$, trimethoprim/sulfamethoxazole $(1.25 / 23.75 \mu \mathrm{g})$, linezolid $(30 \mu \mathrm{g})$, teicoplanin $(30 \mu \mathrm{g})$, vancomycin $(30 \mu \mathrm{g})$, erythromycin $(15 \mu \mathrm{g})$, clindamycin $(2 \mu \mathrm{g})$.The plates were incubated at $35^{\circ} \mathrm{C}$ for $24 \mathrm{hrs}$. The diameter of the zone of inhibition was compared according to Clinical and Laboratory Standard Institute guidelines. ${ }^{11}$ The recommended breakpoints for the $30 \mu \mathrm{g}$ cefoxitin disc used to detect mecA-mediated resistance in S. aureus were as follows resistant $<21 \mathrm{~mm}$ and sensitive $>22 \mathrm{~mm} .{ }^{5}$ All the Staphylococcus aureus isolates showing resistance to erythromycin, were subjected to disc induction test (D test) to study inducible clindamycin resistance. $D$ test was performed by placing the erythromycin disc at a distance of $15 \mathrm{~mm}-20 \mathrm{~mm}$ from the clindamycin disc. A truncated or blunted clindamycin zone of inhibition $(>21 \mathrm{~mm})$ towards erythromycin disc indicates inducible resistance.(Fig. 1) Staphylococcus aureus ATCC 29213 was used as reference strain for the standardization of antibiotic susceptibility testing.

RESULTS: A total of 252 isolates of S. aureus were obtained from different clinical samples of indoor patients of the hospital. Pus and wound swabs accounted for the majority of isolates $168(66.6 \%)$, followed by respiratory specimen 31 (12.3\%), blood cultures $27(10.7 \%)$, urine $20(7.9 \%)$ and body fluids 6 (2.3\%). Out of 252 isolates, 86 (34.1\%) were methicillin resistant Staphylococcus aureus (MRSA) and the remaining 166 (65.8\%) isolates were methicillin sensitive Staphylococcus aureus (MSSA). Maximum isolation of MRSA (38.5\%) was from pus samples, followed by respiratory samples (35.4\%). Figure 2 shows the antibiotic susceptibility data of all the Staphylococcus aureus isolates. None of the MRSA isolates were sensitive to penicillin and ampicillin. All the isolates of Staphylococcus aureus were sensitive to vancomycin, teicoplanin and linezolid.

Amongst MRSA isolates, $52.2 \%$ showed clindamycin resistance, of which constitutive resistance was observed in $28(32.5 \%)$ isolates and inducible in 17(19.7\%) isolates. MS phenotype was observed in $13(15.1 \%)$ MRSA isolates. In MSSA, constitutive and inducible clindamycin resistance was observed in $18(10.8 \%)$ and $8(4.8 \%)$ isolates respectively while MS phenotype was seen in $42(25.3 \%)$ MSSA isolates. 


\section{ORIGINAL ARTICLE}

DISCUSSION: MRSA is a global phenomenon. In the present study, the prevalence rate of MRSA was $34.1 \%$ which is in accordance with other studies from India. $9,13,2,12$. However, some studies have reported alarmingly high rates to the tune of $80 \% .14,8$ Various studies have shown that results of cefoxitin disc diffusion are in concordance with the results of PCR for mecA gene. ${ }^{15,2}$ However, the results of our study could not be confirmed by molecular methods. The limitation of use of cefoxitin is that it detects only MRSA with mecA mediated resistance, although non mecA mediated resistance is a rare occurrence. ${ }^{5,3}$

MRSA shows multi resistance properties. This study also showed that the MRSA isolates were significantly less sensitive to common anti-staphylococcal antibiotics as compared to MSSA isolates. Similar findings have been reported by other studies as well. ${ }^{13}, 16$ In this study, resistance to antibiotics like gentamicin, ciprofloxacin, erythromycin and trimethoprim/sulfamethoxazole was found to be significantly high in MRSA isolates, therefore these antibiotics should not be used for empirical therapy. However, antibiotics like netilmicin, levofloxacin, tetracycline and clindamycin have shown relatively better susceptibility profile in MRSA isolates and thus can be clinically promising. In the present study, susceptibility to ciprofloxacin was low in both MRSA (9.3\%) and MSSA (19.8\%) isolates. On the other hand, levofloxacin sensitivity was seen in $59.3 \%$ and $83.7 \%$ of MRSA and MSSA isolates respectively. In this study, netilmicin also showed excellent susceptibility in $69(80.2 \%)$ MRSA isolates which goes well with other studies. ${ }^{17,9}$ Though vancomycin is the drug of choice for MRSA isolates but we should keep our mind open to use various alternatives before considering vancomycin as the first choice against all MRSA infections. A combination of various antimicrobial agents may also prove to be beneficial to treat resistant infections.

In the present study, inducible clindamycin resistance was observed in $19.7 \%$ and $4.8 \%$ of MRSA and MSSA isolates respectively. This is in concordance with other studies reported earlier. ${ }^{18,19}$ Moreover, both the inducible (19.7\% Vs $4.8 \%$ ) and constitutive (32.5\% Vs 10.8\%) resistance phenotypes were found to be significantly higher in MRSA isolates as compared to MSSA $(\mathrm{P}<0.0001$ and $\mathrm{P}<0.0001$ respectively) which is again in concordance with other studies. ${ }^{13,20}$

Clindamycin serves as one of the very few therapeutic alternatives available for MRSA especially causing skin and soft tissue infection, but expression of inducible resistance to clindamycin by positive $\mathrm{D}$ test limits the effectiveness of this drug. However, CLSI recommendations regarding D test also suggest inclusion of a comment that "this isolate is presumed to be resistant based on detection of inducible clindamycin resistance. Clindamycin may still be effective in some patients." ${ }^{21}$ This comment is important, as relatively minor infections such as cellulitis caused by Dtest positive S. aureus strains (which can be MRSA or MSSA) will respond adequately. However, for sepsis, pneumonia, osteomyelitis and other serious invasive $\mathrm{S}$. aureus infections, even the small risk of emergence of resistance indicated by a positive D-test result generally should lead to avoidance of clindamycin, or prompt change from clindamycin to another agent to which the isolate is susceptible. ${ }^{22}$

CONCLUSION: Clinical microbiology laboratories should consider routine screening (D test) for inducible clindamycin resistance in erythromycin resistant isolates to prevent the possibility of clindamycin treatment failure especially in MRSA. Additionally, robust antimicrobial stewardship and strengthened infection control measures are required to prevent spread and reduce emergence of resistance. 


\section{ORIGINAL ARTICLE}

\section{REFERENCES:}

1. Hsu LY, Wijaya L,Tan BH. Management of health care associated methicillin resistant Staphylococcus aureus. Expert Rev Anti Infect Ther 2005; 3:893-905.

2. Mathews A A, Thomas M, Appalaraju B, Jayalakshmi J. Evaluation and comparison of tests to detect methicillin resistant Staphylococcus aureus. Indian J Patho Microbiol 2010; 53(1):7982.

3. Broekema NM, Van TT, Monson TA, Marshall SA, Warshauer DM. Comparison of Cefoxitin and Oxacillin Disk Diffusion Methods for Detection of mecA-Mediated Resistance in Staphylococcus aureus in a Large-Scale Study. J Clin Microbiol 2009; 47(1) :217-219.

4. Nakatomi Y and Sugiyama J. A rapid latex agglutination assay for the detection of penicillin binding protein. Microbiology and Immunology 1998; 42:739-43.

5. CLSI. Performance standards for antimicrobial susceptibility testing; $17^{\text {th }}$ informational supplement. CLSI Wayne, PA, 2007. M 100-S17.

6. Felten AB, Grandry PH, Lagrange, Casin I. Evaluation of three techniques for detection of lowlevel methicillin-resistant Staphylococcus aureus (MRSA): a disk diffusion method with cefoxitin and moxalactam, the Vitek 2 system, and the MRSA-screen latex agglutination test. J Clin Microbiol 2002; 40:2766-2771.

7. Mimica MJ, Berezin EN, Carvalho RLB, Mimica LMJ, Safad MAP, Schneider E, and Caiaffa-Filho HH. Detection of methicillin resistance in Staphylococcus aureus isolate from pediatric patients: is the cefoxitin disk diffusion test accurate enough? Braz J Infect Dis 2007; 11:415417.

8. Verma S, Joshi S, Chitnus V, Hemvani N, Chitnis D. Growing problems of methicillin resistant staphylococcus -Indian Scenario. Indian J Med Sci 2000; 54:535-40.

9. Rajaduraipendi K, Mani KR, Panneerselvam K, Mani M, Bhaskar M and Manikandan. Prevalence and antimicrobial susceptibility pattern of methicillin resistant Staphylococcus aureus. A multi centre study. Indian Journal of Medical Microbiology 2006; 24(1):34-8.

10. Baird D. Staphylococcus. Cluster forming gram positive cocci. Mackie and McCartney Practical Medical Microbiology. $4^{\text {th }}$ ed. Vol. 2.1996:245-58.

11. CLSI. Performance standards for antimicrobial susceptibility testing; $22^{\text {nd }}$ informational supplement. CLSI. Wayne, PA, 2012. M100-S22.

12. Pai V, Rao VI, Rao SP. Prevalence and Antimicrobial susceptibility pattern of Methicillin resistant Staphylococcus aureus Isolates at a Tertiary Care Hospital in Mangalore, South India Journal of laboratory physicians 2010; 2(2): 82-84.

13. Saikia L, Nath R, Choudhury B, Sarkar M. Prevalence and antimicrobial susceptibility pattern of methicillin resistant Staphylococcus aureus in Assam. Indian J Crit Care Med 2009; 13(3):156-158.

14. Mehta AA, Rodrigues CC, Kumar RR, Rattan AA, Sridhar HH, Mattoo VV , Ginde VV. A pilot programme of MRSA surveillance in India. (MRSA Surveillance Study Group), J Postgrad Med 1996; 42:1-3.

15. Anand KB, Agrawal P, Kumar S, Kapila K. Comparison of cefoxitin disc diffusion test, oxacillin screen agar and PCR for mecA gene for detection of MRSA. Indian J Med Microbiol 2009; 27(1):27-9. 
16. Methicillin resistant Staphylococcus aureus (MRSA) in India. Prevalence and susceptibility pattern. Indian Network for surveillance of Antimicrobial Resistance (INSAR) group, India. Indian J Med Res 2013; 137:363-369.

17. Amruthkishan K. Upadhya, Sunilkumar Biradar .Methicillin resistant Staphylococcus aureus in a tertiary care hospital in north-east Karnataka: evaluation of the antibiogram. Current Research in Medicine and Medical Sciences 2011;1(1): 1-4.

18. Rahabar M, Hajia M. Inducible clindamycin resistance in Staphylococcus aureus. A cross sectional report. Pak J Biol Sci 2007; 10:189-92.

19. Yilmaz G, Aydin K, Iskender S, Caylan R, Koksal I. Detection and prevalence of inducible clindamycin resistance in Staphylococci. J Med Microbio 2007; 56:342-5.

20. Gadepalli R, Dhawan B, Mohanty S, Kapil A, Das BK and Chaudhry R. Inducible clindamycin resistance in clinical isolates of Staphylococcus aureus. Indian J Med Res 2006; 123: 571-573.

21. NCCLS (CLSI). Performance standards for antimicrobial susceptibility testing. NCCLS (CLSI). Wayne, PA, 2004. M100-S14.

22. McGehee RF, Barrett F, Finland M. Resistance of Staphylococcus aureus to lincomycin, clindamycin and erythromycin. Antimicrob Agents Chemother 1968; 8:392-397.
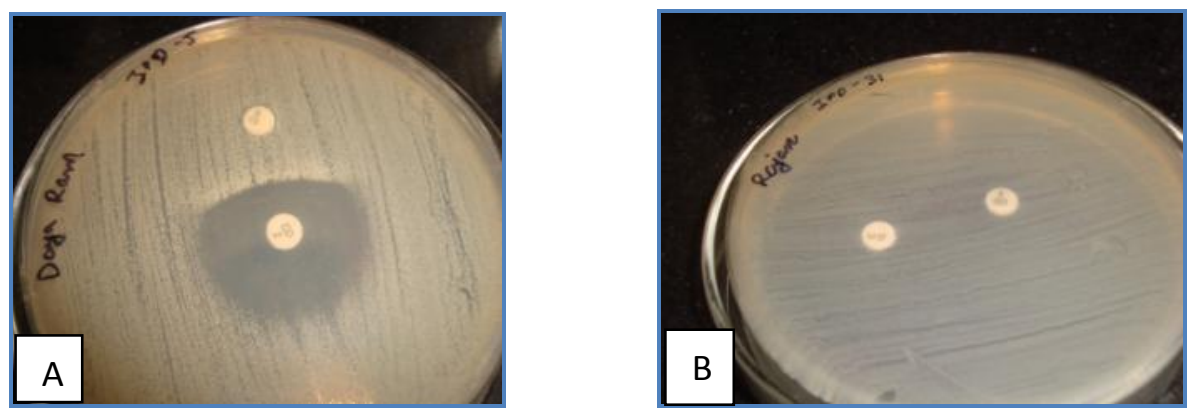

Fig 1: Disc induction test (D test). A) Erythromycin resistant and Clindamycin sensitive Staphylococcus aureus isolate giving D shaped zone of inhibition around clindamycin with flattening towards erythromycin disc suggestive of inducible phenotype. B) Staphylococcal isolate resistant to both erythromycin and clindamycin suggestive of constitutive phenotype.

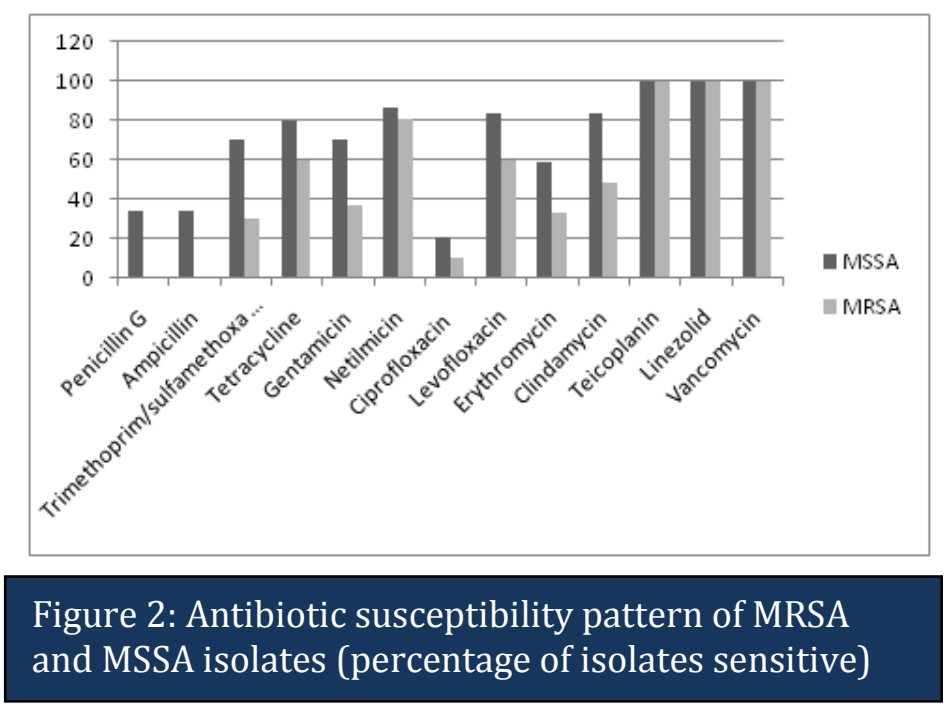




\section{ORIGINAL ARTICLE}

\section{AUTHORS:}

1. Maninder Kaur

2. Satish Gupte

3. Prerna Aggarwal

4. Ashwini Manhas

5. Manju Bala

\section{PARTICULARS OF CONTRIBUTORS:}

1. Assistant Professor, Department of Microbiology, Gian Sagar Medical College and Hospital, Ram Nagar, Rajpura Dist., Patiala.

2. Professor and Head, Department of Microbiology, Gian Sagar Medical College and Hospital, Ram Nagar, Rajpura Dist., Patiala.

3. Associate Professor, Department of Microbiology, Gian Sagar Medical College and Hospital, Ram Nagar, Rajpura Dist., Patiala.
4. Assistant Professor, Department of Microbiology, Gian Sagar Medical College and Hospital, Ram Nagar, Rajpura Dist., Patiala.

5. Assistant Professor, Department of Microbiology, Gian Sagar Medical College and Hospital, Ram Nagar, Rajpura Dist., Patiala.

\section{NAME ADDRESS EMAIL ID OF THE}

\section{CORRESPONDING AUTHOR:}

Dr. Maninder Kaur,

H.No. 103, Block-E,

Rishi Appartment, Sec 70, Mohali.

Email - drmaninderk@yahoo.co.in

Date of Submission: 28/09/2013.

Date of Peer Review: 30/09/2013.

Date of Acceptance: 02/10/2013.

Date of Publishing: 08/10/2013 